\title{
New Research on the Complex Interaction of Sleep and ADHD
}

\author{
Margaret D. Weiss ${ }^{1} \cdot$ Stephanie G. Craig ${ }^{2,3}$ - Gregory Davies ${ }^{3}$ • \\ Larry Schibuk $^{3} \cdot$ Mark Stein $^{4}$
}

Published online: 23 April 2015

(C) Springer International Publishing AG 2015

\begin{abstract}
Children with attention deficit hyperactivity disorder (ADHD) display higher rates of sleep problems such as bedtime resistance, sleep onset difficulties, night awakenings, difficulties with morning awakenings, sleep disordered breathing and daytime sleepiness. There has been a dramatic increase in new research of clinical significance to provide clinicians with the opportunity to better understand the association and possible causal relationships between sleep and ADHD. The objective of this paper is to provide an update on this literature over the past 3 years by examining any aspect of childhood ADHD and sleep and how it may inform clinical practice. As suggested by multiple lines of evidence, results from this review show that at the group level, there is a bidirectional impact of sleep and ADHD on each other and that both sleep and ADHD impairments may stem from common pathways. As hoped, effective intervention for either sleep or ADHD impacts both disorders thus opening the door to better treatment that goes beyond core symptoms to quality of life and functioning.
\end{abstract}

Keywords Attention deficit hyperactivity disorder (ADHD) . Sleep disordered breathing - Sleep onset difficulties .

Neurobehavioural $\cdot$ Neurocognitive

This article is part of the Topical Collection on Sleep and Psychological Disorders

Margaret D. Weiss

margaret.weiss@icloud.com

University of British Columbia, Vancouver, BC, Canada

2 Simon Fraser University, Vancouver, BC, Canada

3 Weiss ADHD Care in Vancouver, Vancouver, BC, Canada

4 University of Washington and Seattle Children's Hospital, Seattle, WA, USA

\section{Background}

Sleep disturbances and disorders are one of the most common comorbidities or conditions associated with attention deficit hyperactivity disorder (ADHD) [1]. Reviews of sleep and ADHD to date have indicated that there appears to be an overlap between symptoms and features of ADHD and the neurobehavioural and neurocognitive effects of sleep disorders $[2,3]$.

The relationship between ADHD and sleep has been well documented in systematic reviews $[2,4,5]$ and meta-analyses $[6,7]$. Disturbed sleep can mimic ADHD in individuals referred for symptoms of ADHD [8]. Children with ADHD display higher rates of sleep problems such as bedtime resistance, sleep onset difficulties, night awakenings, difficulties with morning awakenings, sleep disordered breathing and daytime sleepiness than non-ADHD controls according to subjective ratings and sleep diaries completed by parents $[6$, 9]. On actigraphy, children with ADHD displayed increased sleep onset latency, less true sleep time on actigraphy monitoring, excessive daytime sleepiness as measured by a standard test compared to non-ADHD controls, and lower sleep efficiency on polysomnography [6]. Rates of sleep problems vary depending on demographics, the type of measurement (i.e. subjective vs. objective), and have been cited as being as high as $70 \%$ of youth with ADHD [10].

There is a complex association between difficulties with sleep and ADHD, which is difficult to unravel due to overlapping symptoms and impairments, how and when children are evaluated, and a variety of methodological factors. ADHD may impact self-regulation and the developmental skills necessary to entrain circadian rhythms. Difficulties with sleep impact attention, inhibition, self-regulation and executive function in children with and without ADHD but have a greater cumulative impact on ADHD youth [11]. Both sleep 
disorders and ADHD may be related by similar etiologies, specifically disruption in dopaminergic and melatoninergic pathways. Increased understanding of these mechanisms will better inform the management of ADHD and sleep problems. Although it has long been observed that assessment of sleep requires an assessment for $\mathrm{ADHD}$, more recently, it has been recommended that an assessment for ADHD requires evaluation of sleep [2].

There remains a number of concerns when evaluating the relationship between ADHD and sleep. Prevalence rates of specific sleep problems differ depending on several factors, including ADHD subtype, psychiatric comorbidities and medication use. For example, children with the inattentive subtype in one study displayed fewer night-time sleep problems than those with the combined subtype [12]. This requires further replication, and it is unclear if it is the subtype or severity of ADHD that is driving the relationship. In addition, despite being the first line of treatment for ADHD, methylphenidate and amphetamine stimulants have been shown to be associated with a greater number of sleep difficulties such as increased sleep onset latency and decreased duration of sleep [1], with decreased sleep time associated with increasing dose [13]. Melatonin has been shown to be effective in the reduction of sleep latency problems both for children not medicated [14] and medicated with stimulant medication [15].

Cortese et al. [16] conducted a review of the evidence between sleep and alertness in children with ADHD, controlling for potential confounding factors. They found 13 relevant studies, most of which looked at small samples. These authors concluded that children with ADHD had higher daytime sleepiness, more movements in sleep and higher apneahypopnea indices. They noted that epidemiological studies would be needed to assess the mechanisms underlying the association between sleep, alertness and ADHD in the general population using rigorous and standardized assessment criteria.

There are methodological issues that limit our ability to research the association of sleep and ADHD, or the attempt to assign causal inferences. At this point in time, there is robust research that clearly indicates a strong association of sleep problems with ADHD [1] in children and adolescents. As yet, there is only a paucity of studies examining sleep in adults with ADHD and there may be important developmental differences $[17,18]$. Nonetheless, our understanding in children of some causal relationships has increased markedly over the past decade. We know that stimulants can cause insomnia and frequently exacerbate it when starting a medication trial or when the dose is increased [9]. Furthermore, we know that sleep deprivation causes or compounds difficulty with attention. We anticipate that both ADHD and sleep disorders may represent a final common pathway emerging out of common neurobiological deficits and environmental factors. For example, disruptions in dopamine and melatonin metabolism would be expected to impact both sleep and ADHD. We also do not know if the dysregulation associated with ADHD 'causes' a developmental difficulty with entrainment of circadian rhythms. Night to night variability may exacerbate this, and variability in all settings is a hallmark of environments that predispose someone toward ADHD. We know that there are a minority of children who present with ADHD who may actually have a primary sleep disorder, but far more common are children who have both ADHD and sleep problems. We also know that certain sleep problems, such as obstructive sleep apnea, are associated with increased risk of ADHD, and successful treatment may reduce ADHD symptoms in a minority of individuals [19]. It has, for example, often been noted that the behavioural presentation of an ADHD child is not unlike the behaviour of an overtired child. There is suggestive evidence that ADHD is associated with distinct circadian rhythm patterns, such as the tendency to have a delayed sleep phase (i.e., being a night owl), but again, we do not understand the causal nature of this association $[11,20]$.

\section{Objectives}

Since the last major review of ADHD and sleep [21], there has been considerable new research to provide clinicians with the opportunity to better understand the association and possible causal relationships between sleep and ADHD. The objective of this paper is to provide an update on this literature and how it may inform clinical practice.

We make the assumption that sleep and ADHD are interdependent in multiple ways but that a better understanding of the nature of this relationship may allow clinical intervention leading to improvement in both conditions. Sleep disorders and ADHD may lead to a vicious cycle in which insomnia leads to (or exacerbates) ADHD, and ADHD (and its treatment) further leads to worsening insomnia. If this was to be the case, then understanding of both conditions might lead to greater improvement than would be obtained with a focus on either condition alone.

\section{Methods}

Recent and relevant publications were located using the keywords "ADHD" and "sleep" with PubMed, Google Scholar and PsycINFO. Selected articles included quantitative research published since 2012 that examined any aspect of childhood ADHD and sleep and excluded redundant case studies. Reviews were also excluded from the primary search. The selected articles and methodologies are displayed in Table 1. 


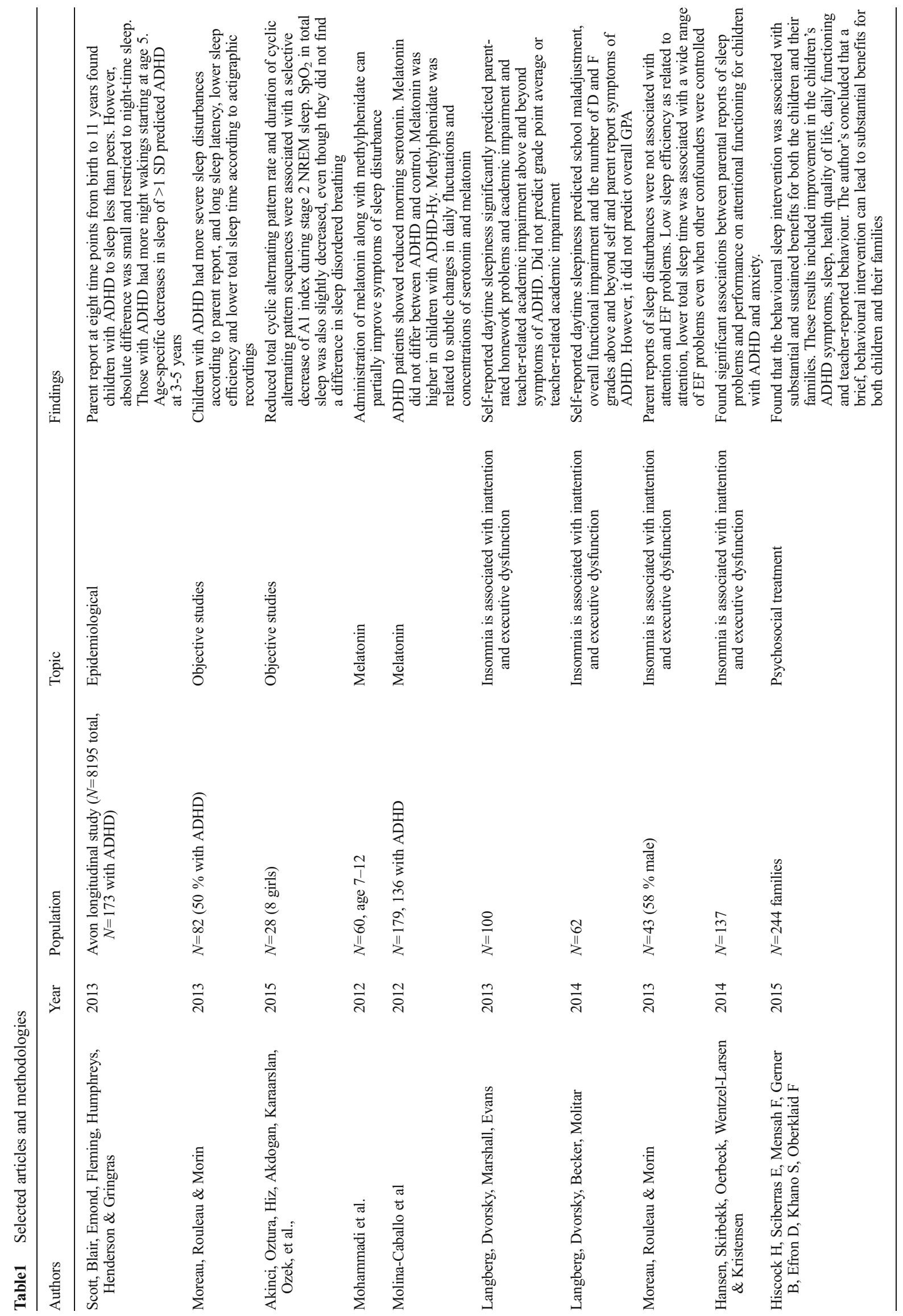




\section{Results}

\section{Epidemiological Associations of Sleep Disorders and ADHD}

Current research has largely replicated the conclusions of earlier studies, providing robust evidence confirming the association between sleep and ADHD. Scott and colleagues [22] followed 8195 children from birth to 11 years with eight follow-up time points. In this study, they found children with ADHD slept less than their peers due to later bedtimes, although the absolute difference was small and restricted to nighttime sleep. They found that those children with ADHD had more night-waking starting at age 5. Interestingly, agespecific decreases in sleep duration measured by parent questionnaires of more than one standard deviation were predictive of an ADHD diagnoses. Likewise, in a sample of youth ( $N=$ 137, age 7-13), Hansen and colleagues [23] also found significant associations between parental reports of sleep problems and performance on attention measures for children with ADHD and comorbid anxiety. We can therefore now say that there is clinical and epidemiological evidence indicating that children presenting with either ADHD or sleep problems will be at greater risk for the other disorder.

\section{Objective Studies}

The first landmark review of the relationship between ADHD and sleep [24] noted a conundrum in which subjective parent reports frequently noted sleep disturbance as a complaint, while there was very little objective evidence of alterations in sleep architecture on polysomnograms. Later, authors hypothesized that this inconsistency might be explained by night to night variability in sleep which would not be detected in a polysomnogram done in one or, at most, two nights [25]. In addition, it may be that polysomnograms may be insensitive to naturalistic factors that trigger sleep impairment in the home environment but not in the lab setting, such as ambient noise, stress related to homework or a parent that is not sleeping. The most recent study of sleep architecture has now found evidence of abnormality in the microstructure of sleep that is not found in the macrostructure of sleep [26]. The authors found reduced total cyclic alternating pattern rate and duration of cyclic alternating pattern sequences were associated with a selective decrease of A1 index during stage 2 NREM sleep. $\mathrm{SpO}_{2}$ in total sleep was also slightly decreased, even though they did not find a difference in sleep disordered breathing.

Early studies showed actigraphic changes in children with ADHD, demonstrating sleep onset insomnia (SOI), decreased sleep duration, sleep variability and sleep efficiency [20, 27, 28]. Actigraphy has, therefore, been a particularly useful tool, which provides somewhat more objective evidence than parent report while remaining naturalistic enough to be able to capture the sleep impairments that occur in the homes of children with ADHD. In 2014, Moreau [29] replicated previous actigraphic studies comparing children with ADHD and controls on both parental and actigraphic measures of sleep. Parental reports, as expected, indicated more severe sleep disturbances. Actigraphy demonstrated longer sleep onset latency, lower sleep efficiency and lower total sleep time. Of note, in this study, there were no differences in sleep measured by actigraphy between children on a stable medication dose and untreated children. Of even more interest is that only the subgroup of children with comorbid psychiatric symptoms (i.e., learning disability, communication disorder, oppositional defiant disorder or generalized anxiety disorder) differed from the control group. The authors concluded that clinicians should systematically attend to sleep disturbances in children with ADHD, particularly when other psychiatric symptoms are also present as these children would be at even greater risk than children with ADHD alone.

\section{Melatonin}

In healthy individuals, the melatonin rhythm is closely associated with the sleep cycle and this relationship has been shown to be disrupted in children with ADHD [30]. In individuals with ADHD and insomnia, there is a delay of nocturnal melatonin onset and differences in melatonin rhythm waveform [31]. This has led to research on possible alterations in melatonin or other neurotransmitters in children with ADHD and insomnia. The demonstration that exogenous melatonin can be used as a sleep aid for children with ADHD [32] further increased interest in understanding the basic biology of regulation of sleep and the pathophysiology of ADHD. Mohammadi and colleagues [33] replicated this finding, and their results were consistent with previous research. In a sample of 60 youths with ADHD between the ages of 7 and 12, melatonin along with methylphenidate led to improvement in symptoms of sleep disturbance. The replication of previous studies $[14,32]$ strengthened their findings. The designs of these three randomized trials looking at melatonin as a treatment for insomnia in children with ADHD are all quite different, but nonetheless come to the same conclusion. If melatonin is a fairly robust method of intervention for ADHD insomnia, we need a better explanation of the biological underpinnings of this relationship.

Molina-Carballo and colleagues [34] investigated the effect of chronic administration of prolonged release methylphenidate on daily variations in blood serotonin (5-HT), melatonin and on the excretion of the 6-sulphatoxy-melatonin (the primary metabolite of melatonin) in urine. In a sample of 179 children (mean age $=9.70$ years, $\mathrm{SD}=2.55$ ), 136 of which met criteria for ADHD as per the DSM-IV, blood samples were drawn at 09:00 and 20:00, and urine was collected at 09:00 and 21:00. Participants with ADHD took prolonged release 
methylphenidate and were reassessed after approximately 4 months. The 43 children who did not have ADHD had a higher 5-HT concentration in the morning as compared to ADHD patients. Baseline serum melatonin did not differ between children with ADHD and controls; however, when examined further, baseline serum melatonin was higher in children with ADHD hyperactive type. The use of methylphenidate was related to subtle changes in daily fluctuations in which melatonin was lower in the morning and slightly higher in the evening. However, the article failed to relate these results to a decrease in sleep onset delay. Further research would be needed to further delineate how stimulants impact melatonin and how melatonin impacts circadian levels of the neurotransmitters involved in ADHD.

\section{Insomnia is Associated with Inattention and Executive Dysfunction}

Several recent studies focused on the effects of sleep on academic related outcomes. The first study found that in a sample of middle school children $(N=100$, mean age $=11.91$ years $)$ self-reported daytime sleepiness significantly predicted parent-rated homework problems, and academic impairment as well as teacher-reported academic impairment. Importantly, these relationships were significant above and beyond the impact of ADHD symptoms on these outcomes [35]. Similar results were found for college age students. Self-reported daytime sleepiness predicted school maladjustment, overall functional impairment and the number of $\mathrm{D}$ and $\mathrm{F}$ grades above and beyond self and parent report symptoms of ADHD. However, it did not predict overall grade point average [36].

We know that executive function deficits are common in youth with ADHD, but not specific to the disorder. We also know that difficulties with organization are a major predictor of adult outcome in their own right [37]. Results from population-based studies also suggest a relationship between sleep disturbances and cognitive functioning [38]. In a sample of 43 children (mean age $=10.1$ years), Moreau [39] did not find that parental reports of sleep disturbances were associated with attention and executive functioning problems. However, they did find that objectively measured lower sleep efficiency was associated with increased variability in attention and that lower total sleep time was associated with a wide range of executive functioning problems as reported by parents. Likewise, Hansen and colleagues [23] found an association between parental reports of sleep problems and performance on attentional function in children with both ADHD and anxiety.

Most recently, an interesting study [40] further explored the relationship between sleep and attention by examining whether poor sleep was a risk factor for attention problems in both children with ADHD and typically developing children. The authors found that while sleep problems impacted alerting and executive attention in all children, executive attention in children with ADHD may be particularly vulnerable to the negative effects of poor sleep. The authors note that these findings highlight the importance of ensuring all children and especially children with ADHD are getting good quality sleep to optimize attention.

\section{Psychosocial Treatment}

The first evidence that sleep hygiene may be a possible treatment for abnormal circadian rhythms was found in the Weiss et al. [32] double-blind, placebo-controlled study of melatonin for sleep onset insomnia in children with ADHD on stimulants. The intervention for these children was gated such that only children who did not respond to sleep hygiene went on to be randomized to melatonin treatment. In this sample, sleep hygiene alone led to remission of SOI to less than $30 \mathrm{~min}$ in $42 \%$ of the sample. The definition of a sleep hygiene responder was that they no longer met inclusion criteria of SOL $>$ $60 \mathrm{~min}$. The authors concluded that a combination of sleep hygiene and melatonin is likely to improve initial insomnia without the recourse of hypnotics.

Hiscock and colleagues [41] have published the first randomized, controlled trial of a brief sleep hygiene intervention in its own right. The intervention consisted of two face-to-face fortnightly (i.e., 2 weeks) consultations with a trained clinician. In the first consultation, the clinician i) assessed the child's sleep problems; ii) elicited parental goals for sleep; iii) provided information on sleep, sleep cycles and sleep hygiene; and iv) formulated a behavioural sleep management plan tailored to the child. Parents were asked to complete a sleep diary between the first and second visit. On the second visit and follow-up telephone call, the clinician reviewed the sleep diary, reinforced strategies, and helped to troubleshoot any problems. The authors found that the behavioural sleep intervention was associated with substantial and sustained benefits for both the children and their families. These results included improvement in the children's ADHD symptoms, sleep, health, quality of life, daily functioning and teacherreported behaviour. The authors' concluded that a brief, behavioural intervention can lead to substantial benefits for both children and their families.

\section{Discussion}

ADHD is a neurobehavioral disorder whose impact is not confined to the school day and, in fact, may represent a $24-\mathrm{h}$ disorder associated with impaired nocturnal and diurnal arousal [42]. In recent research, the importance of night to night variability and effects on attention has been highlighted. The role of circadian rhythm sleep disorders causing or exacerbating ADHD have been demonstrated, linking hyperarousal in 
the evening and attention impairment during the day. ADHD and sleep are not just comorbid, but the day and night manifestations of underlying physiological and neuropsychiatric deficits.

In 2012, Owens and colleagues [43] described several areas worthy of further research. The authors were working from a theoretical framework in which it was hypothesized that an enhanced understanding of the complex mechanisms regulating sleep promotion, wakefulness and attention might contribute new insights regarding the core impairments in either disorder. Among other things, they suggested we needed to better understand how alterations in circadian rhythm contribute to ADHD and explore evidence-based behavioural interventions for sleep disorders in ADHD patients.

The following year, the same authors provided a consensus statement on evidence-based clinical recommendations for intervention in sleep and ADHD stating that both behavioural techniques and melatonin were indicated as interventions for sleep problems in ADHD [21]. It was clear, even then, that we needed treatment strategies to optimize sleep in ADHD and to mitigate the added impact of the impairment caused by insomnia and excessive daytime sleepiness to the cognitive and behavioural impairments of ADHD. It was also clear that characteristic ADHD dysregulation was driving the deficits in entraining circadian rhythms in children with ADHD and thus contributing to eveningness. In the same year, Kooij [44] demonstrated that this was not only true for children but was also consistent at all stages of development.

Recent scientific advances help to clarify the conundrum identified by Corkum [24] as to why sleep was such an important complaint by patients, in the face of a failure to demonstrate objective differences in sleep between ADHD and controls, as polysomnographic studies failed to identify night-tonight variability, and in natura sleep impairment. Indeed, failure to show polysomnographic differences between ADHD and control subjects did not mean that patients were complaining of something that was not there but that early methods of 'objective' assessment of sleep were inadequate to capture the objective differences in sleep in ADHD patients.

In this paper, we have been able to describe new research that has successfully addressed many of the questions raised by the sleep working group 3 years ago $[2,45]$. We have a new body of literature using epidemiological, objective, case control and randomized controlled intervention trials of melatonin and behaviour management - all refining our understanding of the relationship between ADHD and sleep. Epidemiologically, we have established age-specific differences in sleep duration measured by parent questionnaires of more than 1 standard deviation were predictive of ADHD diagnosis. Objectively, refined polysomnograms not only examined sleep architecture but also have been able to identify microstructural differences in sleep between ADHD and controls.
In 2013, we had level 1 evidence, more than two doubleblind, placebo-controlled trials [14, 32], using different research methodologies in different populations to demonstrate that exogenous melatonin could be used as an intervention to regulate sleep in individuals with ADHD. We knew sleep hygiene alone could impact initial insomnia in children with ADHD and that melatonin could further augment that effect in those children who did not achieve remission with sleep hygiene alone [15]. Even more interesting, this relationship held up in the reverse direction. Van der Heijden and colleagues [14] showed that not only was it possible to alter sleep through behavioural interventions but that regulating sleep with melatonin led to improvements in sleep behaviour. We continue to replicate and further elaborate on the robust and bidirectional relationship between sleep hygiene and melatonin. Mohammadi and colleagues [33] replicated previous randomized control trials of melatonin for ADHD insomnia using yet another research design.

New evidence suggests that methylphenidate, a first-line intervention for ADHD, may also impact melatonin levels. Molina-Carballo and colleagues [46] found that administration of methylphenidate was related to subtle changes in the daily fluctuation of melatonin in which melatonin was lower in the morning and slightly higher in the evening in children with ADHD. This is a very interesting finding that needs further study, especially since these changes in fluctuations in melatonin did not translate into what would be an expected improvement in initial insomnia.

Multiple studies using different methodologies and emerging out of different areas have clearly established that insomnia and daytime sleepiness predict of cognitive, academic and executive function impairment $[23,36,39]$. This has led to public health interventions such as delaying the start of the school day as a way of mitigating sleep deprivation and optimizing learning $[47,48]$.

Most recently and long overdue is the first randomized controlled study of a manualized behavioural intervention specifically targeting sleep in children with ADHD [41]. This study established that the sleep intervention was associated with substantial and sustained benefits. The children receiving this intervention had improvement in ADHD symptoms, although the target of intervention was sleep. They also showed improved behaviour, health-related quality of life and daily functioning, all of which go well beyond the core symptoms targeted by previous interventions for ADHD. In addition, the parents also showed improvement with fewer days late for work.

This brings us back full circle to where we started. Parents had always told us that their ADHD children were struggling with sleep and overtired. Children with ADHD had long been observed to resist going to bed, complaining that they 'could not turn their thoughts off'. Since the clinical and research community began to appreciate that these patient complaints 
were both a real and serious source of impairment, it has become possible to develop effective and evidence-based interventions that target both sleep and ADHD. In 2012, we were aware that diagnostic assessment had to address both sleep and ADHD and that intervention for both disorders was necessary to optimize the outcome of either one. The last 3 years have allowed us to refine exactly why this is true and how to intervene. Future research directions will be able to exploit new technologies and methods to allow us to marry objective measurements with naturalistic settings using homebased polysomnography. Mobile technology (e.g. tablet and cell phone)-based methods of actigraphy have made it possible to obtain objective methods of monitoring sleep at home, not only collecting large amounts of data but also allowing us to use actigraphy as part of intervention. Future studies of sleep and ADHD will bring together the best of these epidemiological, objective, subjective and physiological methods.

\section{Conclusion}

Five years ago, the idea that there was a complex association between ADHD and sleep was a hypothesis. As suggested by multiple lines of evidence, it is clear that at the group level, there is a bidirectional impact of sleep and ADHD on each other and that both sleep and ADHD impairments may stem from common pathways. As hoped, effective intervention for either sleep or ADHD impacts both disorders thus opening the door to better treatment that goes beyond core symptoms to quality of life and functioning. It is still an open question whether treatment of both day (ADHD) and night (sleep) abnormalities in alertness leads to a more than additive improvement. New research on the complex associations between sleep and ADHD has and will continue to lead to better clinical interventions and improved patient outcomes.

\section{Compliance with Ethics Guidelines}

Conflict of Interest Margaret D. Weiss has received speaker and consultant honoraria from Eli Lilly, Purdue, and Shire and Janssen.

Stephanie G. Craig, Gregory Davies and Larry Schibuk declare that they have no conflict of interest.

Mark Stein has received grants from Shire, Pfizer and Alcobra and is on the advisory board of Alcobra.

Human and Animal Rights and Informed Consent This article does not contain any studies with human or animal subjects performed by any of the authors.

\section{References}

1. Stein MA, Weiss M, Hlavaty L. ADHD treatments, sleep, and sleep problems: complex associations. Neurotherapeutics. 2012;9(3): 509-17.

2. Cortese S, Brown TE, Corkum P, Gruber R, O'Brien LM, Stein M, et al. Assessment and management of sleep problems in youths with attention-deficit/hyperactivity disorder. J Am Acad Child Adolesc Psychiatry. 2013;52(8):784-96.

3. Owens J, Gruber R, Brown T, Corkum P, Cortese S, O'Brien L, et al. Future research directions in sleep and ADHD: report of a consensus working group. J Atten Disord. 2013;17(7):550-64.

4. Hysing M. Review: recommendations for the assessment and management of sleep disorders in ADHD. Evid Based Ment Health. 2014;17(1):22.

5. Barrett JR, Tracy DK, Giaroli G. To sleep or not to sleep: a systematic review of the literature of pharmacological treatments of insomnia in children and adolescents with attention-deficit/hyperactivity disorder. J Child Adolesc Psychopharmacol. 2013;23(10):640-7.

6. Cortese S, Faraone SV, Konofal E, Lecendreux M. Sleep in children with attention-deficit/hyperactivity disorder: meta-analysis of subjective and objective studies. J Am Acad Child Adolesc Psychiatry. 2009;48(9):894-908.

7. Sedky K, Bennett DS, Carvalho KS. Attention deficit hyperactivity disorder and sleep disordered breathing in pediatric populations: a meta-analysis. Sleep Med Rev. 2014;18(4):349-56.

8. Owens JA. The ADHD and sleep conundrum: a review. J Dev Behav Pediatr. 2005;26(4):312-22.

9. Stein MA, Mendelsohn J, Obermeyer WH, Amromin J, Benca R. Sleep and behavior problems in school-aged children. Pediatrics. 2001;107(4):E60.

10. Engelhardt CR, Mazurek MO, Sohl K. Media use and sleep among boys with autism spectrum disorder, ADHD, or typical development. Pediatrics. 2013;132(6):1081-9.

11. Gruber R, Fontil L, Bergmame L, Wiebe ST, Amsel R, Frenette S, et al. Contributions of circadian tendencies and behavioral problems to sleep onset problems of children with ADHD. BMC Psychiatry. 2012;12:212.

12. Mayes SD, Calhoun SL, Bixler EO, Vgontzas AN, Mahr F, Hillwig-Garcia J, et al. ADHD subtypes and comorbid anxiety, depression, and oppositional-defiant disorder: differences in sleep problems. J Pediatr Psychol. 2008;34(3):328-37.

13. Santisteban JA, Stein MA, Bergmame L, Gruber R. Effect of extended-release dexmethylphenidate and mixed amphetamine salts on sleep: a double-blind, randomized, crossover study in youth with attention-deficit hyperactivity disorder. CNS Drugs. 2014;28(9):825-33.

14. Van der Heijden KB, Smits MG, Van Someren EJ, Ridderinkhof KR, Gunning WB. Effect of melatonin on sleep, behavior, and cognition in ADHD and chronic sleep-onset insomnia. J Am Acad Child Adolesc Psychiatry. 2007;46(2):233-41.

15. Weiss MD, Wasdell MB, Bomben MM, Rea KJ, Freeman RD. Sleep hygiene and melatonin treatment for children and adolescents with ADHD and initial insomnia. J Am Acad Child Adolesc Psychiatry. 2006;45(5):512-9.

16. Cortese S, Konofal E, Yateman N, Mouren MC, Lecendreux M. Sleep and alertness in children with attention-deficit/hyperactivity disorder: a systematic review of the literature. Sleep. 2006;29(4): 504-11.

17. Surman CB, Roth T. Impact of stimulant pharmacotherapy on sleep quality: post hoc analyses of 2 large, double-blind, randomized, placebo-controlled trials. J Clin Psychiatry. 2011;72(7):903-8.

18. Surman CB, Thomas RJ, Aleardi M, Pagano C, Biederman J. Adults with ADHD and sleep complaints: a pilot study identifying 
sleep-disordered breathing using polysomnography and sleep quality assessment. J Atten Disord. 2006;9(3):550-5.

19. Marcus CL, Moore RH, Rosen CL, Giordani B, Garetz SL, Taylor $\mathrm{HG}$, et al. A randomized trial of adenotonsillectomy for childhood sleep apnea. N Engl J Med. 2013;368(25):2366-76.

20. Gruber R, Sadeh A, Raviv A. Instability of sleep patterns in children with attention-deficit/hyperactivity disorder. J Am Acad Child Adolesc Psychiatry. 2000;39(4):495-501.

21. Owens J, Gruber R, Brown T, Corkum P, Cortese S, O'Brien L, et al. Future research directions in sleep and adhd report of a consensus working group. J Atten Disord. 2013;17(7):550-64.

22. Scott N, Blair PS, Emond AM, Fleming PJ, Humphreys JS, Henderson J, et al. Sleep patterns in children with ADHD: a population-based cohort study from birth to 11 years. J Sleep Res. 2013;22(2):121-8.

23. Hansen BH, Skirbekk B, Oerbeck B, Wentzel-Larsen T, Kristensen $\mathrm{H}$. Associations between sleep problems and attentional and behavioral functioning in children with anxiety disorders and ADHD. Behav Sleep Med. 2014;12(1):53-68.

24. Corkum P, Moldofsky H, Hogg-Johnson S, Humphries T, Tannock R. Sleep problems in children with attention-deficit/hyperactivity disorder: impact of subtype, comorbidity, and stimulant medication. J Am Acad Child Adolesc Psychiatry. 1999;38(10):1285-93.

25. Cohen-Zion M, Ancoli-Israel S. Sleep in children with attentiondeficit hyperactivity disorder (ADHD): a review of naturalistic and stimulant intervention studies. Sleep Med Rev. 2004;8(5):379-402.

26. Akinci G, Oztura I, Hiz S, Akdogan O, Karaarslan D, Ozek H, Akay A: Sleep structure in children with attention-deficit/hyperactivity disorder. Journal of Child Neurology 2015.

27. Stein MA, Blondis TA, Schnitzler ER, O'Brien T, Fishkin J, Blackwell B, et al. Methylphenidate dosing: twice daily versus three times daily. Pediatrics. 1996;98(4 Pt 1):748-56.

28. Radek K. Are actiwatch recordings useful in the diagnosis and treatment of attention deficit disorder and hyperactivity. J Sleep Disord Ther. 2013;2(148):2167-0277.10001.

29. Moreau V, Rouleau N, Morin CM. Sleep of children with attention deficit hyperactivity disorder: actigraphic and parental reports. Behav Sleep Med. 2014;12(1):69-83.

30. Van Veen MM, Kooij JJ, Boonstra AM, Gordijn MC, Van Someren EJ. Delayed circadian rhythm in adults with attention-deficithyperactivity disorder and chronic sleep-onset insomnia. Biol Psychiatry. 2010;67(11):1091-6.

31. Novakova M, Paclt I, Ptacek R, Kuzelova H, Hajek I, Sumova A. Salivary melatonin rhythm as a marker of the circadian system in healthy children and those with attention-deficit/hyperactivity disorder. Chronobiol Int. 2011;28(7):630-7.

32. Weiss M, Wasdell M, Bomben M, Rea K, Freeman R. Sleep hygiene and melatonin treatment for children and adolescents with ADHD and initial insomnia. J Am Acad Child Adolesc Psychiatry. 2006;45(5):512-9.

33. Mohammadi MR, Mostafavi SA, Keshavarz SA, Eshraghian MR, Hosseinzadeh P, Hosseinzadeh-Attar MJ, et al. Melatonin effects in methylphenidate treated children with attention deficit hyperactivity disorder: a randomized double blind clinical trial. Iran J Psychiatry. 2012;7(2):87-92.
34. Molina-Carballo A, Naranjo-Gómez A, Uberos J, Justicia-Martínez F, Ruiz-Ramos M-J, Cubero-Millán I, et al. Methylphenidate effects on blood serotonin and melatonin levels may help to synchronise biological rhythms in children with ADHD. J Psychiatr Res. 2013;47(3):377-83.

35. Langberg JM, Dvorsky MR, Marshall S, Evans SW. Clinical implications of daytime sleepiness for the academic performance of middle school-aged adolescents with attention deficit hyperactivity disorder. J Sleep Res. 2013;22(5):542-8.

36. Langberg JM, Dvorsky MR, Becker SP, Molitor SJ. The impact of daytime sleepiness on the school performance of college students with attention deficit hyperactivity disorder (ADHD): a prospective longitudinal study. J Sleep Res. 2014;23(3):318-25.

37. Barkley RA, Fischer M. Predicting impairment in major life activities and occupational functioning in hyperactive children as adults: self-reported executive function (EF) deficits versus EF tests. Dev Neuropsychol. 2011;36(2):137-61.

38. Bos SC, Gomes A, Clemente V, Marques M, Pereira A, Maia B, et al. Sleep and behavioral/emotional problems in children: a population-based study. Sleep Med. 2009;10(1):66-74.

39. Moreau V, Rouleau N, Morin CM. Sleep, attention, and executive functioning in children with attention-deficit/hyperactivity disorder. Arch Clin Neuropsychol. 2013;28(7):692-9.

40. Waldon J, Vriend J, Davidson F, Corkum P: Sleep and attention in children with ADHD and typically developing peers. J. Atten. Disord. 2015.

41. Hiscock H, Sciberras E, Mensah F, Gerner B, Efron D, Khano S, et al. Impact of a behavioural sleep intervention on symptoms and sleep in children with attention deficit hyperactivity disorder, and parental mental health: randomised controlled trial. BMJ. 2015;350:h68.

42. Stein MA, Weiss M, Hlavaty L. ADHD treatments, sleep, and sleep problems: complex associations. Neurotherapeutics. 2012;9(3): 509-17.

43. Owens J, Gruber R, Brown T, Corkum P, Cortese S, O’Brien L, et al. Future research directions in sleep and ADHD report of a consensus working group. J Atten Disord. 2013;17(7):550-64.

44. Kooij JJ, Bijlenga D. The circadian rhythm in adult attention-deficit/hyperactivity disorder: current state of affairs. Expert Rev Neurother. 2013;13(10):1107-16.

45. Owens J, Gruber R, Brown T, Corkum P, Cortese S, O'Brien L, et al. Future research directions in sleep and ADHD: report of a consensus working group. J Atten Disord. 2012;17(7):550-64.

46. Molina-Carballo A, Naranjo-Gomez A, Uberos J, Justicia-Martinez F, Ruiz-Ramos MJ, Cubero-Millan I, et al. Methylphenidate effects on blood serotonin and melatonin levels may help to synchronise biological rhythms in children with ADHD. J Psychiatr Res. 2012;47(3):377-83.

47. Boergers J, Gable CJ, Owens JA. Later school start time is associated with improved sleep and daytime functioning in adolescents. J Dev Behav Pediatr: JDBP. 2014;35(1):11-7.

48. Owens JA, Belon K, Moss P. Impact of delaying school start time on adolescent sleep, mood, and behavior. Arch Pediatr Adolesc Med. 2010;164(7):608-14. 